\title{
CHAT BOTS APPLICATION AND EFFICIENCY FOR PATIENT SELF-MANAGEMENT SUPPORT
}

\author{
Sadrieh Hajesmaeel Gohari ${ }^{1}$, Zahra Karbasi ${ }^{2}$, Sharareh R. Niakan Kalhori*3
}

1: PhD student of Health Information Management, Department of Health Information Management, School of Allied Medical Sciences, Tehran University of Medical Sciences (TUMS), Tehran, Iran

2: PhD student of Health Information Management, Department of Health Information Management, School of Allied Medical Sciences, Tehran University of Medical Sciences (TUMS), Tehran, Iran

3: PhD of Medical Informatics, Department of Health Information Management, School of Allied Medical Sciences, Tehran University of Medical Sciences, Tehran, Iran

Correspondence:

Tel: +98. 2188982886, Fax:+98. 2188983037, E-mail: sh-rniakank@sina.tums.ac.ir

\section{TYPE OF ARTICLE: CONFERENCE ABSTRACT}

\begin{abstract}
Introduction: Patients who have problems in language and communication need new technology as training to improve the condition in their daily lives. One of the interesting fields of virtual reality is an interactive artificial intelligence computer program called a chat bot, which can be used to communicate with people and exchange information. People can encounter with them on social networks, mobile or websites. A chat bot simulates human conversation with patients to check up and help them to take their medication. The bot asks the right question about symptoms and type of patient disease. This study was aimed to review the effective use of chat bots for patient training and consulting.

Methods: This review article was done through PubMed, with "chat bot" and "health" for keywords. We analyzed obtained articles on the basis of objective research.

Results: In this research, we look to the use of chat bots for different disease management in published articles. The results of the collected data indicate that chat bots have been used in neurodegenerative diseases like Parkinson's. The use of this technology in health that runs on a smartphone is studied in Australia. Results showed that a chat bot can be effective in speech and communication therapy for a patient's daily life; it also can play a role as an advisor and sponsor.

Conclusion: Studies showed positive results of using chat bots in a clinical field. Chat bots can comprehend and understand users'; therefore, they can be considered as an option to support chronic disease self-management, particularly for those who need more integrative requirements. Because of the importance of therapeutic chat bots, usability evaluation of them for medication adherence is recommended.

KEYWORDS: Chat bot, Virtual reality, Human-Computer interaction, Patient
\end{abstract}

\footnotetext{
Abstracts of First National Congress of Medical Informatics, Mashhad, Iran, February 2017

(C) 2017 The Authors. This is an open access article under the terms of the Creative Commons Attribution-NonCommercialNoDerivs License, which permits use and distribution in any medium, provided the original work is properly cited, the use is non-commercial and no modifications or adaptations are made.
} 